\title{
POLYMOLECULARITY AND MECHANICAL PROPERTIES OF CELLULOSE ACETATE
}

\author{
By Arnold M. Sookne and Milton Harris ${ }^{1}$
}

\begin{abstract}
The tensile strengths, ultimate elongations, and folding endurances of films prepared from a series of cellulose acetate fractions and blends were studied. When the mechanical properties are plotted against the intrinsic viscosities (or relative weight-average degrees of polymerization), the results for the fractions and different blends fall on separate curves. In contrast, when the mechanical properties are plotted against the number-average degrees of polymerization, the results for the fractions and all of the blends fall approximately on a single curve for each property. The results are shown to be qualitatively consistent with the hypothesis that the mechanical properties of blends are the weight-averages of the properties of their components. The results emphasize the importance of the determinations of the number-average degree of polymerization in studying commercial polymolecular materials.
\end{abstract}

\section{CONTENTS}

I. Introduction

II. Results and discussion

1. Mechanical properties as a function of intrinsic viscosity and number-average degree of polymerization _...

2. Additivity of mechanical properties

III. Conclusion _ _

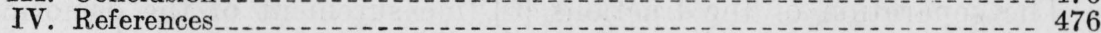

\section{INTRODUCTION}

High polymers are in general polymolecular; that is, heterogeneous with respect to molecular size. The problem of the influence of the degree of polymolecularity on the mechanical properties of such materials is an important one, and it has received considerable study in recent years. The detailed results of these investigations are reviewed elsewhere $[1,2,3]^{2}$. The procedure generally followed in these studies was to fractionate a polymer with respect to molecular size, and compare the mechanical properties of the fractions with those of blends. There has been substantial agreement among the more recent investigators that fractionated cellulosic materials are superior in mechanical properties to blends of the same intrinsic viscosity; i. e., the same weight-average molecular weight $[1,4,5]$. In particular, it has been found that the presence of material of low

1 Research Associates at the National Bureau of Standards representing the Textile Foundation.

2 Figures in brackets indicate literature references at the end of this paper. 


\section{8

molecular weights exerts a harmful influence on the mechanical properties of blends. These results appear to be unambiguous only for unoriented or slightly oriented specimens; it is quite possible that other conclusions may be reached when oriented or crystallized materials are considered.

In an approach to this problem some time ago, a large quantity of commercial cellulose acetate was fractionated in this laboratory [6], and some of the mechanical properties of the fractions and their blends were studied [4]. It was found that these mechanical properties were more closely related to the number-average than to the weight-average $D P$ 's ${ }^{3}$ of the samples. However, the $\overline{D P}_{w}$ 's were at that time calculated from viscosity measurements at only one concentration (by means of Staudinger's rule), and the $\overline{D P}_{n}$ 's of the blends were calculated under the assumption that the fractions were perfectly homogeneous. The $\overrightarrow{D P}_{n}$ 's of the fractions have now been determined experimentally by osmotic pressure measurements in acetone solution, and their intrinsic viscosities have been determined in the same solvent [7]. It is the purpose of this discussion to reexamine the data for the mechanical properties of the fractions and blends in the light of these new measurements of $\overrightarrow{D P}_{n}$ and intrinsic viscosity. In addition, examination of the data shows that to a first approximation the mechanical properties of the blends are the weight-averages of the properties of their components; i. e.,

$$
\text { Property blend }=\frac{\Sigma w_{i} P_{i}}{\Sigma w_{i}}
$$

where $w_{i}$ is the weight of the molecular species with a mechanical property $P_{i}$. It will be shown that the experimentally obtained relationships between the mechanical properties and the $D P_{n}$ 's and intrinsic viscosities are all consistent with this relationship.

\section{RESULTS AND DISCUSSION}

The separation of the fractions [6], measurement of the osmotic pressure values and intrinsic viscosities [7], and the preparation and mechanical testing of films of the fractions and blends [4] have been described in detail elsewhere.

\section{MECHANICAL PROPERTIES AS A FUNCTION OF INTRINSIC VIS- COSITY AND NUMBER-AVERAGE DEGREE OF POLYMERIZATION}

Figure 1 shows the tensile strengths of the fractions as a function of their intrinsic viscosities (upper graph) and $\overline{D P}_{n}$ 's (lower). In considering these and subsequent data (figs. 1 to 6 ), the intrinsic viscosity may be taken as a direct measure of $\overline{D P}_{w}$ on the basis of Kraemer's results [8] and because of the linear relationship between $\overline{D P}_{n}$ and $[\eta]$ (intrinsic viscosity) found for the fractions [7]. Because of the uncertainty in the value of Kraemer's constant, the data have been plotted as a function of $[\eta]$ rather than of $\overline{D P}_{w .}{ }^{4}$ The curves have

\footnotetext{
3 The weight-average and number-average degrees of polymerization will henceforth be written $\overline{D P}_{\omega}$ and $\overrightarrow{D P}_{n}$, respectively.

4 The scale of the axis of abscissae in each upper graph of figures 1 to 6 has, however, been so adjusted that it compares directly with the $D P$ axis of the lower graph, assuming $\overline{D P}_{\omega}=230$ [ $\eta$ ] (Kraemer's relationship).
} 


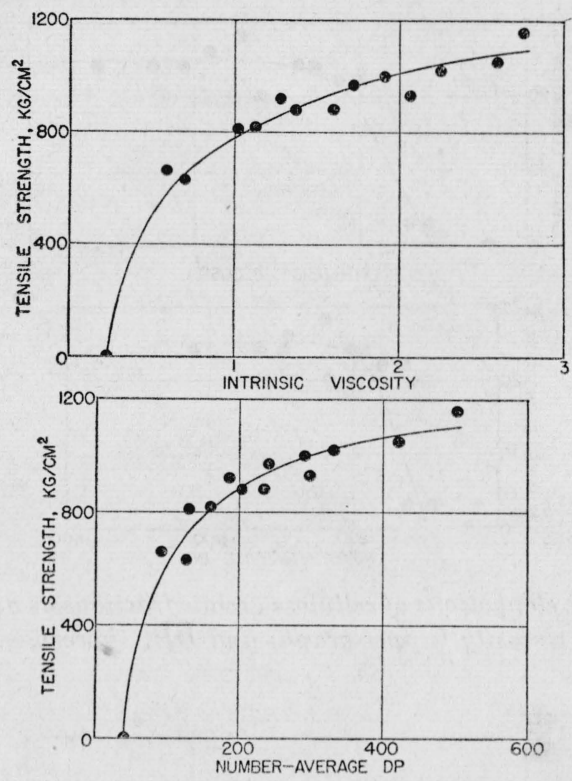

FigURE 1.-Tensile strengths of cellulose acetate fractions as a function of intrinsic viscosity (upper graph) and $\overrightarrow{D P}_{n}$ (lower).

a large slope in the region of small chain length, followed by a gradually decreasing slope which may represent an approach to an asymptotic value for very high $D P$ 's. Similarly shaped curves have been observed for numerous polymers [9]. The fraction of lowest chain length $\left(\overline{D P}_{n}=43\right)$ was so brittle that it would not form a coherent film of sufficient size to be tested by ordinary technics, and it has therefore been assigned a strength of zero. Small fragments of this sample were tested by special technics, however, and a strength as high as $250 \mathrm{~kg} / \mathrm{cm}^{2}$ was obtained. This result suggests that the failure to obtain measurable strengths for this and other samples of very low $D P$ results from the inadequacy of the film casting and testing technics, rather than from the weakness of the sample. Although it is true that such materials have zero strength as far as their practical utilization is concerned, the possibility exists that the curve relating strength to $D P$ would pass through the origin if suitable testing technics were used.

Figure 2 shows the ultimate elongations of the fractions, plotted in the same manner as the tensile strength results. The shapes of the latter curves are generally similar to those for the tensile strength data, except that the approach to a limiting value is completed at a lower $D P$ for the ultimate elongation results. As shown in figure 3, entirely analogous results were obtained for the measurements of the folding endurances of the fractions (made on a Massachusetts Institute of Technology Fold Tester).

In order to study the effect of distribution of chain length on mechanical properties, blends were prepared from pairs of the fractions. The major portion of the work on blends was done with three series, prepared by mixing in various proportions pairs of fractions of 

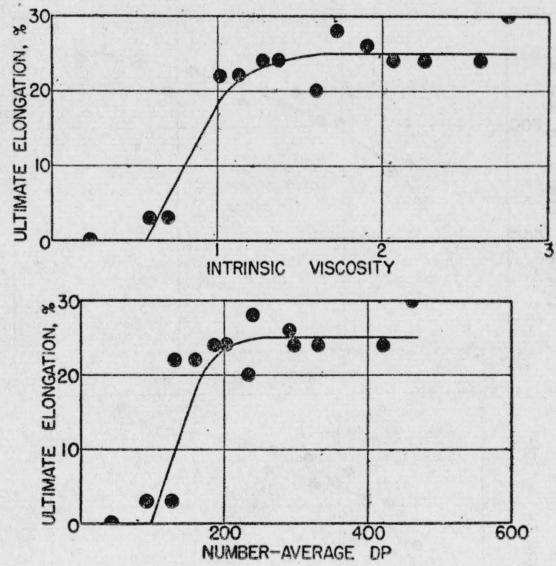

FIGURE 2.-Ultimate elongations of cellulose acetate fractions as a function of intrinsic viscosity (upper graph) and $\overrightarrow{D P}_{n}$ (lower).
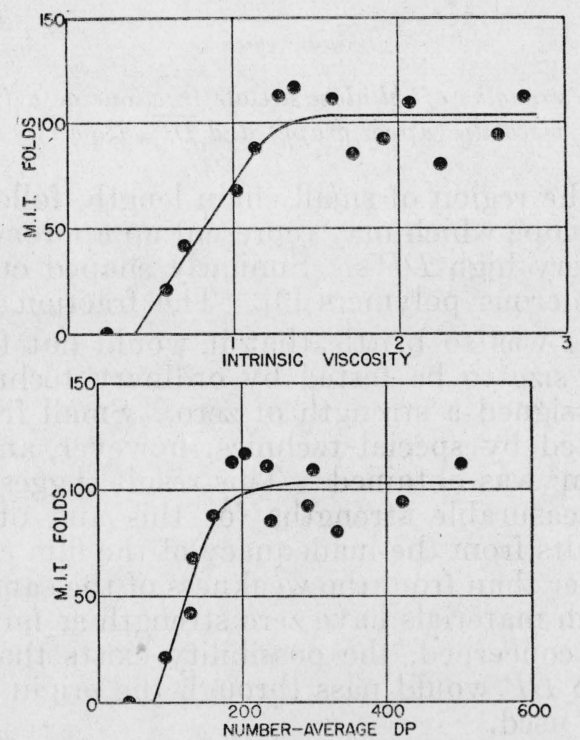

FIGURE 3.-Folding endurances of cellulose acetate fractions as a function of intrinsic viscosity (upper graph) and $\overline{D P}_{n}$ (lower).

the following $\overline{D P}_{n}$ 's: $502+43,502+127$ and $502+204$. (These same pairs of fractions are identified by their intrinsic viscosities of $2.75+.23,2.75+.70$, and $2.75+1.37$, respectively.) A number of miscellaneous blends and the starting material were also studied. The intrinsic viscosities and $\overline{D P}_{n}$ 's of the blends were calculated from 
their known weight compositions and the intrinsic viscosities and $\overline{D P}_{n}$ 's of the fractions. ${ }^{5}$

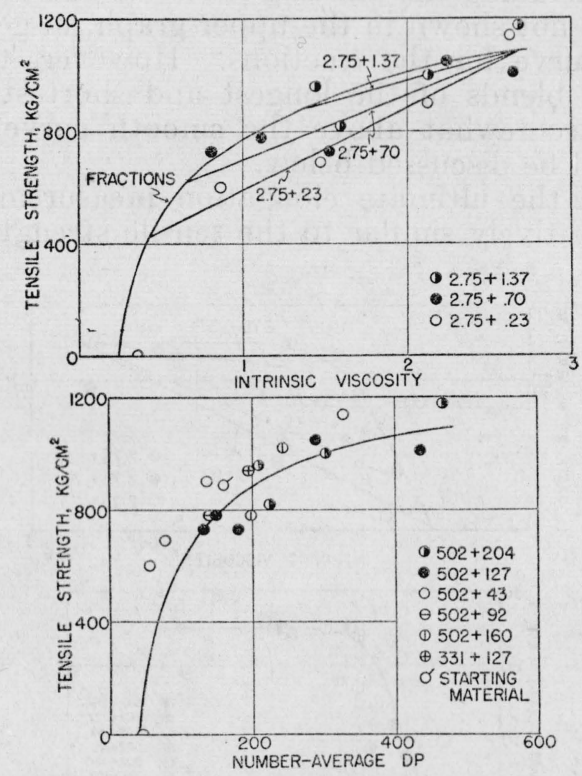

FIGURE 4.-Tensile strengths of blends of cellulose acetate fractions as a function of intrinsic viscosity (upper graph) and $\overrightarrow{D P}_{n}$ (lower).

The curve for the fractions (traced from fig. 1) is included for comparison.

Figure 4 shows the tensile strengths of the blends as a function of both their intrinsic viscosities (upper graph) and $\overline{D P}_{n}$ 's (lower). The respective curves for the fractions, traced from the smooth curves of figure 1, are included for comparison. As shown by the upper graph of figure 4 , the strengths of the blends are in general lower than those of the fractions when the results are plotted against intrinsic viscosity (or relative $\overline{D P}_{w}$ ). Further, the discrepancy between the curve for the fractions and that for a series of blends appears to increase with increasing difference in chain length between the components in a given series. Thus, the open circles, which represent blends of the longest and shortest chain lengths, fall farthest

6 The intrinsic viscosities of the blends were calculated from the formula

$$
[\eta]_{b l e n d}=f_{1}[\eta]_{1}+f_{2}[\eta]_{2},
$$

where $f_{1}$ and $f_{2}$ are the weight fractions in the blend of the components of intrinsic viscosities $[\eta]_{1}$ and $[\eta]_{2}$, respectively. The values of $\overline{D P}_{n}$ for the blends were obtained from the relationship

$$
\overline{D P}_{n_{\text {blend }}}=\frac{1}{\frac{f_{1}}{\overline{D P}_{n_{1}}}+\frac{f_{2}}{\overline{D P}_{n_{2}}}},
$$

where $f_{1}$ and $f_{2}$ are the weight fractions in the blend of the components of $\overline{D P}_{n_{1}}$ and $\overline{D P}_{n_{2}}$, respectively.

Calculated rather than experimental values of $[\eta]$ and $\overline{D P}_{n}$ for the blends were used because of the unquestioned validity of the calculation procedure, and because samples of the original blends sufficient for the experimental measurements were not available. 
below the curve for the fractions. In contrast, when the tensile strengths are plotted against $\overrightarrow{D P}_{n}$ (lower graph, fig. 4), the results for the blends, including the starting material and a number of miscellaneous blends not shown in the upper graph, in general fall closer to the smooth curve for the fractions. However, the open circles (which represent blends of the longest and shortest chain lengths) fall consistently somewhat above the smooth curve. The possible cause for this will be discussed below.

The results of the ultimate elongation measurements on blends (fig. 5) are qualitatively similar to the tensile strength data, but the
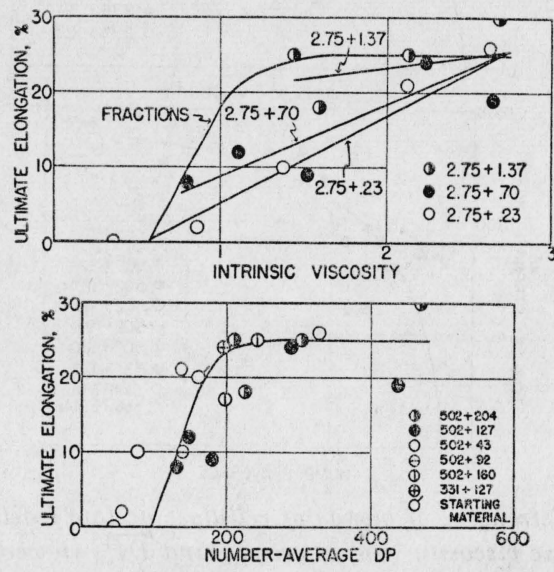

FIgURE 5.-Ultimate elongations of blends of cellulose acetate fractions as a function of intrinsic viscosity (upper graph) and $\overrightarrow{D P}_{n}$ (lower).

The curve for the fractions (traced from fig. 2) is included for comparison.

differences between the upper and lower graphs are more clearly defined. In the upper graph, the curves for the blends of fractions of intrinsic viscosities $2.75+.23$ fall markedly below the smooth curve for the fractions (traced from fig. 2), the results for the series $2.75+.70$ are intermediate, and those for the series $2.75+1.37$ are quite close to the curve for the fractions. In the lower graph, in which the results are plotted against $\overrightarrow{D P}_{n}$, all of the results for the blends fall fairly close to the curve for the fractions; the open circles, however, again consistently fall somewhat above the smooth curve.

The results for the folding endurance measurements (fig. 6) are analogous to those for the ultimate elongations. The differences between the blends and fractions are again large when the results are plotted against intrinsic viscosity. The results for the series $2.75+$ 1.37 appear to fall somewhat above the smooth curve for the fractions, but it is possible that this difference results from the variability inherent in folding endurance measurements rather than any actual superiority of the blends. As shown by the lower graph of figure 6, the results for all the blends once more fall fairly close to the curve for the fractions when $\overline{D P}_{n}$ is taken as the independent variable. Again, however, the results for the series $\overline{D P}_{n}$ 's $502+43$ (open circles) fall slightly above the curve for the fractions. 

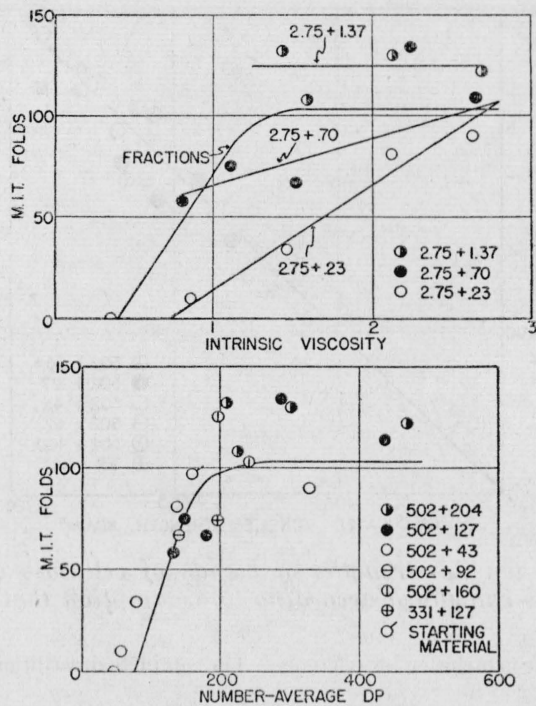

FIgURE 6.-Folding endurances of blends of cellulose acetate fractions as a function of intrinsic viscosity (upper graph) and $\overline{D P}_{n}$ (lower).

The curve for the fractions (traced from fig. 3 ) is included for comparison.

\section{ADDITIVITY OF MECHANICAL PROPERTIES}

The results described above demonstrate that the mechanical properties of blends are more closely related to $\overline{D P}_{n}$ than to $\overline{D P}_{w}$. It can be shown that this closer relationship between mechanical properties and $\overrightarrow{D P}_{n}$ is consistent with two factors: the shape of the curve relating mechanical properties to $D P$ for the fractions, and the fact that the mechanical properties of a blend are the weight-averages of the properties of their components (eq 1). The second point will be considered first.

Examination of the tensile strength data for the blends reveals that if a strength of approximately $260 \mathrm{~kg} / \mathrm{cm}^{2}$ is assigned to the fraction of lowest $D P$, for which no precise experimental value could be obtained, the strength of each blend is the weight-average of the strengths of its components. This is shown in figure 7 , in which the observed strength of each blend has been plotted against the value calculated (according to eq 1) from the weight percentages and strengths of the fractions of which it is composed. A line of 45-degree slope passing through the origin is included in the figure for comparison. The one blend which departs seriously from the line (observed strength, 0 ; calculated strength, 310) was a 95:5 mixture by weight of the fractions of shortest and longest chain lengths, respectively. This blend, like the fraction of lowest $D P$, was too brittle to give a film suitable for testing.

A similar relationship between the observed and calculated ultimate elongations of the blends can also be demonstrated, but because of the lower precision of these results, the agreement between the observed and calculated values is not so good as for the tensile strength measurements. The folding endurances of all the blends except those contain- 


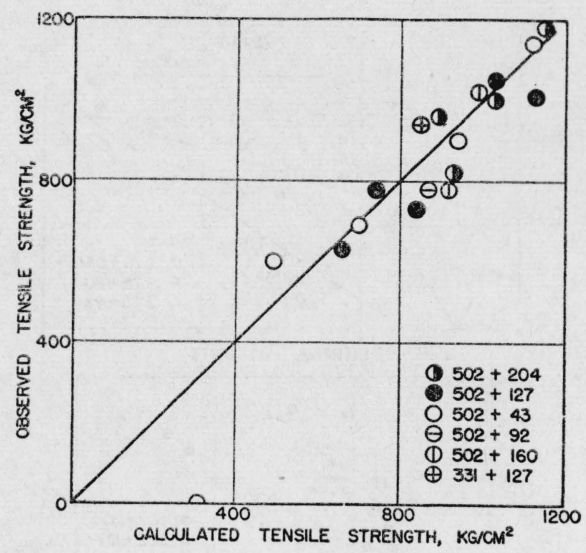

FIGURE 7.-Observed tensile strengths of blends of cellulose acetate fractions as a function of strengths calculated according to assumption that strengths of fractions are additive (eq 1).

The $45^{\circ}$ line is included for reference. For complete description, see text.

ing the fraction of smallest chain length $\left.\overrightarrow{D P}_{n}=43\right)$ are also approximately additive. For the latter blends, the folding endurance values are additive only if the fraction with a $\overline{D P}_{n}$ of 43 is assumed to have a negative folding endurance. This somewhat unreasonable result is, however, consistent with the shape of the curve relating folding endurance to $\overrightarrow{D P}_{n}$ for the fractions (fig. 3), as extrapolation of this curve to a $\overrightarrow{D P}_{n}$ of 43 would lead to a negative folding endurance.

It is now of interest to examine the consequences of applying the concept of additivity of mechanical properties to curves relating such properties to chain length for fractionated materials. For this purpose, the curve shown in figure 8 was chosen. It should be emphasized, however, that the results described below would be qualitatively similar if any of the experimental curves of figures 1 to 3 were used in this discussion. Figure 8 is a theoretical curve calculated from the equations of Sullivan [10], relating strength and fiber length of yarns which are perfectly homogeneous with respect to fiber

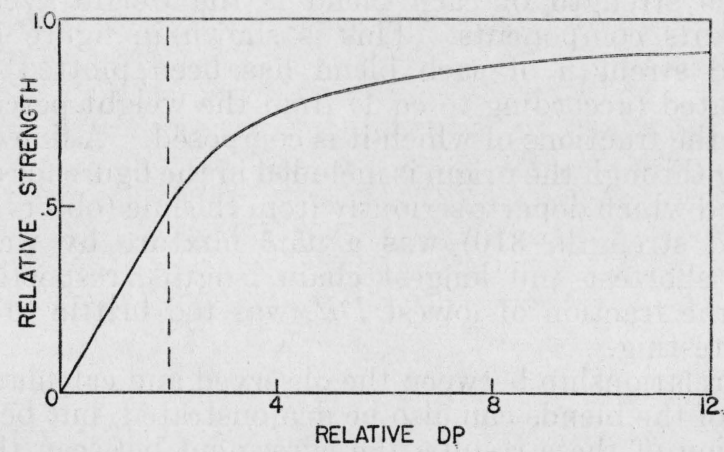

FIGURE 8.-Theoretical curve, relating strength to DP for perfectly homogeneous polymers. 
length. ${ }^{6}$ It differs qualitatively from typical experimental curves only in that it passes through the origin. As suggested above, however, the failure of the experimental strength- $D P$ curves to pass through the origin may well result from technical difficulties in film preparation and testing. In any case, this difference does not seriously affect the conclusions drawn below.

Figure 9 shows the strengths of blends, calculated from the curve of figure 8 , assuming additivity of strength. As in the experimental curves, the strengths have been plotted on both weight- and numberaverage bases. Results for four series of blends have been plotted, representing varying proportions of the following pairs: $4+10,2.5+$ $10,1+10$, and $.01+10$. The numbers have been so chosen that the first three of these approximately represent the three series of blends
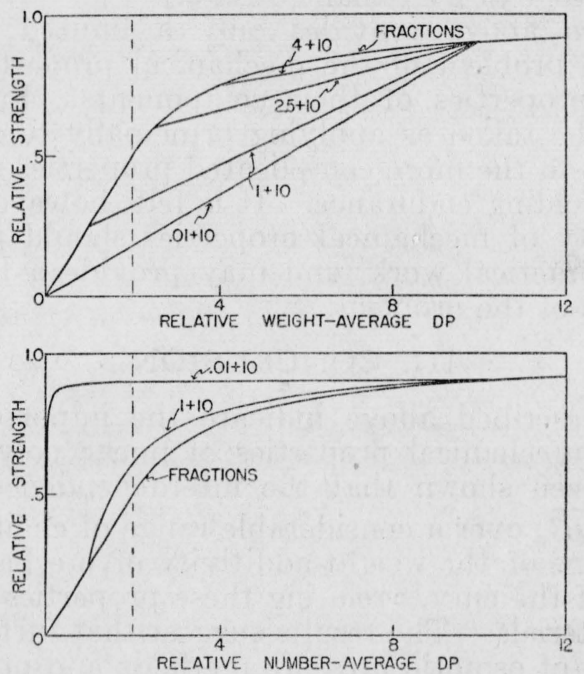

FigURE 9.-Theoretical curves, relating strength to $\overrightarrow{D P}_{w}$ (upper graph) and $\overrightarrow{D P}_{n}$ (lower) for blends of homogeneous polymers.

The curve for the fractions is identical with that given in figure 8. See text for complete explanation.

studied experimentally, and the last is roughly equivalent to blends of the fraction of highest $D P$ and a monomer. As shown by the upper graph of the figure, when the results are plotted on a weightaverage basis, the calculated strengths of the blends fall on straight lines below the curve for the fractions. The extent of the departure from the curve for the fractions increases with the disparity between the chain lengths of the eomponents in a pair. In contrast, when the results are plotted on the number-average basis, the curves for the blends of pairs $4+10$ and $2.5+10$ fall directly on the curve for the fractions. The curve for the pair $1+10$, which corresponds to the

\footnotetext{
${ }^{6}$ According to Sullivan's analysis [10], to the left of the broken line in figure 8, yarn breakage occurs solely by slippage of the fibers. As the strength is contributed solely by frictional forces in this range, it is directly proportional to fiber length. To the right of the broken line, the frictional forces are sufficiently large to cause some fiber breakage, and the strength approaches asymptotically to a maximum value at which all fibers break. The analogy between frictional forces between fibers in a yarn and interchain forces in a film or filament is fairly close, but it is not suggested that the breaking in tension of a film can be explained in terms of this analysis. Thus, the breaking of a film includes a complex process of flow and crystallization, for which there is no counterpart in a yarn. The two processes do, however, contain a number of interesting points of similarity.
} 
experimental blends of the longest and shortest chain lengths, falls slightly above the curve for the fractions, as did the open circles in the lower graphs of figures 4,5 , and 6 . The curve for the pair .01+10, however, falls far above the curve for the fractions, which result is also in accord with experience [3]. Thus, these curves, which merely represent a statement of the weight-additivity of the strength, reproduce all of the features of the experimental data. It is perhaps worth noting that the series $1+10$ (and the corresponding experimental series, as well) contains blends considerably more heterogeneous than the common unplasticized commercial polymers. Thus, some of the blends in this series have a ratio of $\overline{D P}_{w} / \overline{D P}_{n}$ higher than 3 , as compared with a value of about 2 for most ordinary polymers. Even for this series, however, the mechanical properties are much more closely related to $\overline{D P}_{n}$ than to $\overline{D P}_{20}$.

The discussion above provides only a limited and qualitative approach to the problem of the mechanical properties of blends as related to the properties of their components. Further, the conclusions should be taken as applying principally to strength properties, rather than to the more complicated properties such as ultimate elongation and folding endurance. It is felt, however, that the concept of additivity of mechanical properties should prove helpful in some types of practical work, and may provide a basis for a more detailed analysis of the problem.

\section{CONCLUSION}

The results described above indicate the importance of $\overline{D P}_{n}$ in considering the mechanical properties of linear polymolecular polymers. It has been shown that the interdependence of mechanical properties and $\overline{D P}_{n}$ over a considerable range of chain lengths can be explained in terms of the weight-additivity of mechanical properties, and the shape of the curves relating these properties and the $D P$ for fractionated materials. The results suggest that further attention be given to the use of osmotic pressure methods and other methods for determining $\overrightarrow{D P}_{n}$ in industrial work.

\section{REFERENCES}

[1] H. M. Spurlin, Ind. Eng. Chem. 30, 538 (1938).

[2] H. Mark, Paper Trade J. 113, 34 (1941).

[3] H. M. Spurlin, chapter in Cellulose and cellulose derivatives, by Emil Ott (Interscience Publishers, New York, N. Y., 1943) p. 930-42.

[4] A. M. Sookne and M. Harris, J. Research NBS 30, 1 (1943) RP1513.

[5] W. Schieber, Papier-Fabr., Tech.-wiss. T1. 37, 245 (1939).

[6] A. M. Sookne, H. A. Rutherford, H. Mark, and M. Harris, J. Research NBS 29, 123 (1942) RP1490.

[7] A. M. Sookne and M. Harris, J. Research NBS 34, 459 (1945) RP1654.

[8] E. O. Kraemer, Ind. Eng. Chem. 30, 1200 (1938).

[9] H. Mark, chapter in Cellulose and cellulose derivatives, by Emil Ott (Interscience Publishers, New York, N. Y., 1943) p. 990.

[10] R. R. Sullivan, J. Applied Physics 13, 157 (1942).

Washington, December 14, 1944. 
NATIONAL BUREAU OF STANDARDS, WASHINGTON 25, D. C.

Send me the Mathematical Tables marked $\times$ below. I enclose remittance to cover the cost.

\begin{tabular}{|c|c|c|c|c|}
\hline Mark X & Title of publication & $\begin{array}{l}\text { United States and } \\
\text { its possessions, } \\
\text { and countries ex- } \\
\text { tending franking } \\
\text { privilege }\end{array}$ & Other countries & Amount enclosed \\
\hline $\begin{array}{l} \\
\end{array}$ & 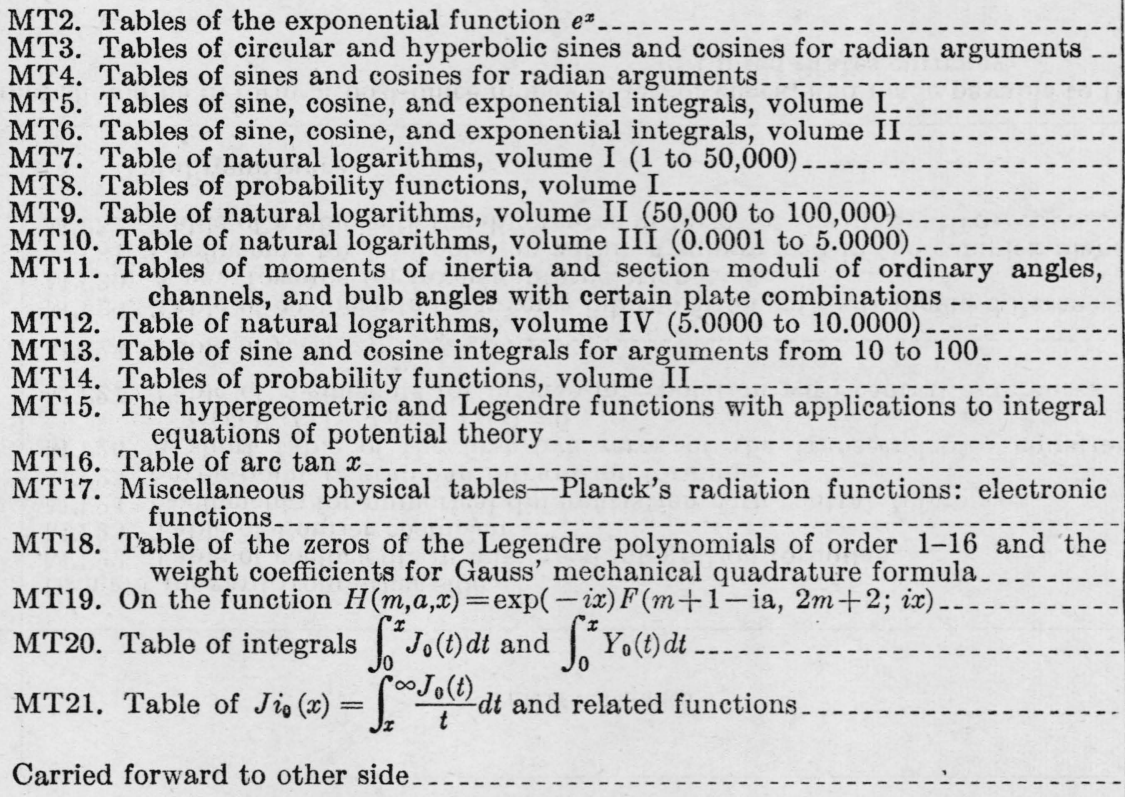 & $\begin{array}{r}\$ 2.00 \\
2.00 \\
2.00 \\
2.00 \\
2.00 \\
2.00 \\
2.00 \\
2.00 \\
2.00 \\
2.00 \\
2.00 \\
2.00 \\
2.00 \\
2.00 \\
2.00 \\
1.50 \\
.25 \\
.25 \\
.25 \\
.25 \\
\end{array}$ & $\begin{array}{r}\$ 2.50 \\
2.50 \\
2.50 \\
2.50 \\
2.50 \\
2.50 \\
2.50 \\
2.50 \\
2.50 \\
2.50 \\
2.50 \\
2.50 \\
2.50 \\
2.50 \\
2.50 \\
1.75 \\
.30 \\
.30 \\
.30 \\
.30\end{array}$ & 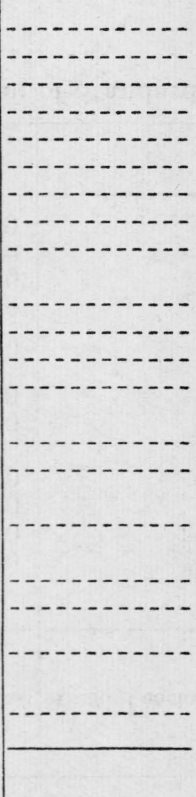 \\
\hline
\end{tabular}




\begin{tabular}{|c|c|c|c|c|}
\hline Mark X & Title of Publication & $\begin{array}{l}\text { United States and } \\
\text { its possessions, } \\
\text { and countries ex- } \\
\text { tending franking } \\
\text { privilege }\end{array}$ & Other countries & Amount enclosed \\
\hline ' & $\begin{array}{l}\text { Brought forward from other side } \\
\text { MT22. Table of coefficients in numerical integration formulae } \\
\text { MT23. Table of Fourier coefficients } \\
\text { MT24. Coefficients for numerical differentiation with central differences } \\
\text { MT25. Seven-point Lagrangian integration formulas } \\
\text { MT26. A short table of the first five zeros of the transcendental equation, } \\
\text { MT27. Table of coefficients for inverse interpolation with central differences } J_{0}(x) Y_{0}(k x)-J_{0}(k x) Y_{0}(x)=0 \\
\text { MT28. Table of } f_{n}(x)=\frac{n !}{(x / 2) n} J_{n}(x) \\
\text { MT29. Table of coefficients for inverse interpolation with advancing differences } \\
\text { MT30. A new formula for inverse interpolation } \\
\text { MT31. Coefficients for interpolation within a square grid in the complex plane. } \\
\text { MT32. Table of coefficients for differences in terms of the derivatives } \\
\quad \text { Total remittance. }\end{array}$ & $\begin{array}{r}\$ 0.25 \\
.25 \\
.25 \\
.25 \\
.25 \\
.25 \\
.25 \\
.25 \\
.25 \\
.25 \\
.25\end{array}$ & $\begin{array}{r}\$ 0.30 \\
.30 \\
.30 \\
.30 \\
\\
.30 \\
.30 \\
.30 \\
.30 \\
.30 \\
30 \\
.30\end{array}$ & \begin{tabular}{|c|}
-2 \\
\\
\end{tabular} \\
\hline
\end{tabular}

Remittance should be in form of post-office money order, or check, and made payable to the order of the "National Bureau of Standards" in United States currency

Send to

Number and Street

City, zone, and State 


\section{MATHEMATICAL TABLES}

Attention is invited to a series of publications prepared by the Project for the Computation of Mathematical Tables conducted by the Federal Works Agency, Work Projects Administration for the City of New York, under the sponsorship of the National Bureau of Standards. The tables which have been made available through the National Bureau of Standards are listed below. A list of other WPA tables obtainable elsewhere will be sent by the Bureau on request.

There is included in this list a publication of the hypergeometric and Legendre functions (MT15). prepared by the Bureau.

MT1. Table of the First Ten Powers of the Integers From 1 to 1000: (1938) VIII+80 pages: heavy paper cover. Out of print.

MT2. TABles of the Exponential. Function $e^{x}$ : The ranges and intervals of the argument and the number of decimal places in the entries are given below:

$\begin{array}{rcc}\text { Range of } x & \text { Interval of } x & \text { Decimals given } \\ -2.5000 \text { to } 1.0000 & 0.0001 & 18 \\ 1.0000 \text { to } 2.5000 & .0001 & 15 \\ 2.500 \text { to } 5.000 & .001 & 15 \\ 5.00 \text { to } 10.00 & .01 & 12\end{array}$

(1939) XV + 535 pages; bound in buckram, $\$ 2.00$.

MT3. Tables op Circular and Hyperbolic Sines and Cosines por Radian Arguments: Con. tains 9 decimal place values of $\sin x, \cos x, \sinh x$, and $\cosh x$ for $x$ (in radians) ranging from 0 to 2 at intervals of 0.0001 . (1939) XVII +405 pages; bound in buckram, $\$ 2.00$.

MT4. Tables of Sines and Cosines por Radian Arguments: Contains 8 decimal place values of sines and cosines for radian arguments ranging from 0 to 25 at intervals of 0.001 . (1940) XXIX +275 pages; bound in buckram, $\$ 2.00$.

MT5. Tables of Sine, Cosine, and Exponential Integrals, Volume I: Values of these functions to 9 places of decimals from 0 to 2 at intervals of 0.0001 . (1940) XXVI+444 pages; bound in buckram, $\$ 2.00$.

MT6. Tables op Sine, Cosine, and Exponential. Integral.s, Volume II: Values of these functions to 9.10 , or 11 significant figures from 0 to 10 at intervals of 0.001 with auxiliary tables. (1940) XXXVII+225 pages: bound in buckram, $\$ 2.00$.

MT7. Table op Natural Logarithms, Volume I: Logarithms of the integers from 1 to 50,0C0 to 16 places of decimals. (1941) XVIII+ 501 pages; bound in buckram, $\$ 2.00$.

MT8. Tables of Probability Functions, Volume I: Values of these functions to 15 places of decimals from 0 to 1 at intervals of 0.0001 and from 1 to 5.6 at intervals of 0.001 . (1941) XXVIII+302 pages; bound in buckram, $\$ 2.00$.

MT9. Table of Natural Logarithms, Volume II: Logarithms of the integers from 50,000 to 100,000 to 16 places of decimals. (1941) XVIII+501 pages; bound in buckram, $\$ 2.00$.

MT10. Table of Natural Logarithms, Volume III: Logarithms of the decimal numbers from 0.0001 to 5.0000 , to 16 places of decimals. (1941) XVIII + 501 pages; bound in buckram, $\$ 2.00$.

MT11. Tables of the Moments of Inertia and Section Moduli op Ordinary Angles, Chane nels, and Bulb Angles with Certain Plate Combinations: (1941) XIII + 197 pages; bound in green cloth. $\$ 2.00$.

MT12. Table of Natural Logarithms, Volume IV: Logarithms of the decimal numbers from 5.0000 to 10.0000 , to 16 places of decimals. (1941) XXII + 506 pages; bound in buckram, $\$ 2.00$.

MT13. TABle of Sine ANd Cosine InTEgrals for Arguments from 10 to 100: (1942) XXXII+185 pages; bound in buckram, $\$ 2.00$.

MT14. Tables of Probability Functions, Volume II: Values of these functions to 15 places of decimals from 0 to 1 at intervals of 0.0001 and from 1 to 7.8 at intervals of 0.001 . (1942) XXI+344 pages; bound in buckram, $\$ 2.00$.

MT15. The Hypergeometric and Legendre Functions With Applications to Integral Equations op Potential Theory. By Chester Snow, National Bureau of Standards. Reproduced from original handwritten manuscript. (1942) VII+319 pages, bound in heavy paper cover. $\$ 2.00$.

MT16. Table of Arc TAN $x$ : Table of inverse tangents for positive values of the angle in radians. Second central differences are included for all entries. $x=[0(.001) 7(.01) 50(.1) 300(1) 2,000(10)$ $10,000 ; 12 D]$ (1942) XXV+169 pages; bound in buckram, $\$ 2.00$.

[Continued on p. 4 of cover] 
MT17. Miscellaneous Physical Tables: Planck`s Radiation Functions (Originally published in the Journal of the Optical Society of America, February 1940); and Electronic Functions. (1941) VII + 58 pages; bound in buckram, $\$ 1.50$.

MT18. Table of the Zeros of the Legendre Polynomials of Order 1-16 and the Weicht Coepricients por Gauss' Mechanical Quadrature Formula. (Reprinted from Bul. Amer. Mathematical Society, October 1942.) 5 pages, with cover, 25 cents.

MT19. ON the Function $H(m, a, x)=\exp (-i x) F(m+1-i a, 2 m+2 ; i x)$; with table of the confluent hypergeometric function and its first derivative. (Reprinted from J. Math. Phys., December 1942.) 20 pages, with cover, 25 cents.

MT20. Table of Intrgrals $\int_{0}^{x} J_{0}(t) d t$ and $\int_{0}^{*} Y_{0}(t) d t$. (Reprinted from J. Math. Phys., May 1943.) 12 pages, with cover, 25 cents.

MT21. Table of $J i_{0}(x)=\int_{x}^{\infty} \frac{J_{0}(t)}{t} d t$ and Related Functions. (Reprinted from J. Math. Phys., June 1943.) 7 pages, with cover, 25 cents.

MT22. Table of Coefficients in Numerical Intrgration Formulae. (Reprinted from J. Math. Phys., June 1943.) 2 pages, with cover, 25 cents.

MT23. Table op Fourier Coefpicients. (Reprinted from J. Math. Phys., Sept. 1943.) 11 pages, with cover, 25 cents.

MT24. Corppicients for Numerical. Difperentiation With Central. Dipferences. (Reprinted from J. Matn. Phys., Sept. 1943.) 21 pages, with cover, 25 cents.

MT25. Seven-Point Lagrangian Intrgration Formulas. (Reprinted from J. Math. Phys., Dec. 1943.) 4 pages, with cover, 25 cents.

MT26. A Short Table of the First Five Zeros of the Transcendental Equation. $J_{0}(x) Y_{4}(k x)-J_{0}(k x) Y_{0}(x)=0$. (Repinted from J. Math. Phys., Dec. 1943.) 2 pages, with cover, 25 cents.

MT27. Table of Coefpicients por Inverse Interpolation With Central Dipperences. (Reptinted from J. Math. Phys., Dec. 1943.) 15 pages, with cover, 25 cents.

MT28. TABLE of $f_{n}(x)=\frac{n !}{(x / 2)^{n}} J_{n}(x)$. (Reprinted from J. Math. Phys., Feb. 1944) 16 pages, with cover, 25 cents

MT29. Table or Cozpricients for Inverse Interpolation With Advancing Difperences (Reprinted from J. Math. Phys., May 1944.) 28 pages, with cover, 25 cents.

MT30. A New Formula for Inverse Intrrpolation. (Reprinted from Bul. Amer. Mathematical Society, Aug. 1944.) 4 pages, with cover, 25 cents.

MT31. Coepricients for Interpolation Within a Square Grid in the Complex Plane. (Re. printed from J. Math. Phys., Aug. 1944). 11 pages, with cover. 25 cents.

MT32. Table op Cozpricients in Terms or the Derivatives. (Reprinted from J. Math. Phys. Nov. 1944.) 4 pages, with cover, 25 cents.

Payment is required in advance. Make remittance payable to the "National Bureau of Standards" and send with order, using the blank form facing page 3 of the cover.

A mailing list is maintained for those who desire to receive announcements regarding' new tables as they become available. 